\title{
Exciton-phonon coupling efficiency in CdSe quantum dots embedded in ZnSe nanowires
}

\author{
S. Bounouar, ${ }^{1,2}$ C. Morchutt, ${ }^{2}$ M. Elouneg-Jamroz,${ }^{1,2}$ L. Besombes,${ }^{1}$ R. André, ${ }^{1}$ E. Bellet-Amalric, ${ }^{2}$ C. Bougerol, ${ }^{1}$ \\ M. Den Hertog, ${ }^{3}$ K. Kheng, ${ }^{2}$ S. Tatarenko, ${ }^{1}$ and J. Ph. Poizat ${ }^{1}$ \\ ${ }^{1}$ CEA-CNRS-UJF group "Nanophysique et Semiconducteurs”, Institut Néel, CNRS-Université Joseph Fourier, 38042 Grenoble, France \\ ${ }^{2}$ CEA-CNRS-UJF group "Nanophysique et Semiconducteurs", CEA/INAC/SP2M, 38054 Grenoble, France \\ ${ }^{3}$ Institut Néel, CNRS-Université Joseph Fourier, 38042 Grenoble, France
}

(Received 23 September 2011; revised manuscript received 25 November 2011; published 19 January 2012)

\begin{abstract}
Exciton luminescence of a CdSe quantum dot (QD) inserted in a ZnSe nanowire is strongly influenced by the dark exciton states. Because of the small size of these QDs (2-5 nm), exchange interaction between hole and electron is highly enhanced and we measured large energy splitting between bright and dark exciton states $(\Delta E \in[4,9.2] \mathrm{meV})$ and large spin-flip rates between these states. Statistics on many QDs showed that this splitting depends on the QD size. Moreover, we measured an increase of the spin-flip rate to the dark states with increasing energy splitting. We explain this observation with a model, taking into account the fact that the exciton-phonon interaction depends on the bright to dark exciton energy splitting, as well as on the size and shape of the exciton wave function. It also has consequences on the exciton line intensity at high temperature.
\end{abstract}

DOI: 10.1103/PhysRevB.85.035428

PACS number(s): 78.67.Lt, 78.55.Et

\section{INTRODUCTION}

Semiconductor nanowires (NWs) have attracted great attention in the past few years, since they hold great promise to become building blocks in tomorrow's nanoscale devices and circuits with vast potential applications ranging from nanoelectronics, ${ }^{1-3}$ optoelectronics (light-emitting diodes, ${ }^{4,5}$ nanolasers ${ }^{6}$ ), and thermoelectrical energy conversion ${ }^{7}$ to biological or chemical sensors. ${ }^{8}$ Most of the NW growth methods allow for the variation of the chemical composition ${ }^{9,10}$ or doping ${ }^{11}$ along the longitudinal or radial directions. This enables the fabrication of semiconductor heterostructures, and more specifically of quantum dots (QDs).

Single QDs have turned out to be excellent candidates for stable and efficient single-photon sources. ${ }^{12-16}$ Within this category, QDs embedded in NWs have already demonstrated single-photon emission, ${ }^{17}$ even at relatively high temperature $(T=220 \mathrm{~K}){ }^{18}$ The quest for efficient and eventually room-temperature QD single-photon sources requires a good understanding of the excitonic dynamics in such systems.

In a QD, exciton states are split by the electron-hole exchange interaction into higher-energy bright exciton states (BS) and lower-energy dark states (DS) ${ }^{19}$ with respective angular momentum of $J_{z}= \pm 1$ and $J_{z}= \pm 2$. In the present system, the energy splitting is relatively large, and has already been measured around $\Delta E=6 \mathrm{meV} .{ }^{20}$ As exchange interaction in semiconductor materials is proportional to the spatial overlap between the electron and hole wave functions, it is strongly enhanced in low-dimensional objects. Values of $\Delta E \in[2,4] \mathrm{meV}$ have been measured in quantum wells ${ }^{21}$ and it has been demonstrated that they can be even higher in quantum wires. ${ }^{22}$ Extremely large splitting was calculated and observed $^{23}$ in colloidal QDs $(\Delta E \in[2,20] \mathrm{meV})$. This is the result of very good confinement of the carriers owing to the small size of the QDs. Effects of the dark states on the QD luminescence become noticeable when $\Delta E \gg k_{B} T$, with $k_{B}$ the Boltzmann constant and $T$ the temperature. ${ }^{20,24,25}$ We show that, under nonresonant pumping, the BS excitonic population leaks toward the DS that recombines nonradiatively, leading to a reduced excitonic light emission compared to the biexcitonic one. Transitions between the BS and DS states are due to hole spin flips assisted by phonons. ${ }^{26}$ The speed of these processes depends essentially on the efficiency of the hole interaction with the phonon reservoir experienced by the QD. ${ }^{27}$

In this paper, we show that the QD size can highly influence the QD phonon spectral density and modify the spin-flip rates between the bright and dark exciton states. It not only has consequences on the exciton intensity at $T=4 \mathrm{~K}$, but also at high temperatures.

The paper is organized as follows. The sample preparation and the setup are presented in Sec. II. In Sec. III, the BS to DS energy splitting $\Delta E$ of several QDs is extracted using temperature-dependent lifetime measurements. In Sec. IV, we present the experimental dependence of the spin-flip rate versus $\Delta E$, and suggest an explanation based on a theoretical model. These results are then used in Sec. V to discuss the exciton to biexciton line intensity ratio as a function of $\Delta E$ and of the temperature.

\section{SAMPLE AND SETUP}

The nanowires are grown by molecular-beam epitaxy using the vapor-liquid-solid technique. ${ }^{28}$ The substrate is GaAs 100 with a $\mathrm{ZnSe}$ buffer. After dewetting of a thin layer of gold at $500{ }^{\circ} \mathrm{C}$, the growth is performed at $410{ }^{\circ} \mathrm{C}$. The nanowires have a diameter of $10 \mathrm{~nm}$ and a length of $400 \mathrm{~nm}$. The wire diameter (around $10 \mathrm{~nm}$ ) is of the order of the bulk exciton Bohr diameter in CdSe $(11 \mathrm{~nm})$, which means that the carriers in the QD are in the strong confinement regime. In order to study single QD, the nanowires are broken and dispersed on a silicon substrate by direct contact. High-resolution transmission electron microscope (TEM) images revealed very small QD sizes, from 2 to $5 \mathrm{~nm}$ in the nanowire direction. ${ }^{29}$ Other TEM experiments coupled to spectroscopy on other samples showed that this growth technique often led to $\mathrm{ZnSe}$ encapsulated QDs. Photoluminescence on as-grown samples is centered around $2.35 \mathrm{eV}$ with a dispersion of $\pm 0.07 \mathrm{eV}$.

The experimental apparatus is a time-resolved microphotoluminescence experiment setup. The excitation source is a 
frequency doubled picosecond Ti sapphire laser emitting at a wavelength of $\lambda=950 / 2=475 \mathrm{~nm}$ (i.e., $2.6 \mathrm{eV}$ ). Nanowires luminescence is detected through a $\delta \lambda=0.04 \mathrm{~nm}$ (i.e., $\delta E=$ $0.2 \mathrm{meV}$ ) resolution spectrometer on a charged coupled device (CCD) camera for spectra or on a low jitter (40 ps) avalanche photodiode (APD) for time-resolved measurements (60 ps time resolution for the whole setup).

\section{QD SIZE EFFECT ON THE EXCHANGE INTERACTION}

Figure 1(a) shows a typical spectrum obtained on a single nanowire. The two transitions correspond to exciton and biexciton (respectively denoted $X$ and $X X$ ) and were identified using cross-correlation techniques. ${ }^{20}$ The biexciton binding energy is measured around $20 \mathrm{meV}$. The four relevant states of a neutral QD are represented on Fig. 1(c). The exciton is split in two states (noted DS and BS) linked by spin-flip transition rates noted $\gamma_{\mathrm{sp} 1}$ and $\gamma_{\mathrm{sp} 2}$. The quantities $\Gamma_{X}$ and $\Gamma_{X X}$ are the radiative recombination rates of the bright exciton and the biexciton, and $\Gamma_{\mathrm{nr}}$ is the nonradiative decay rate of the dark exciton. These quantities have been measured at $T=4 \mathrm{~K}$ on nine
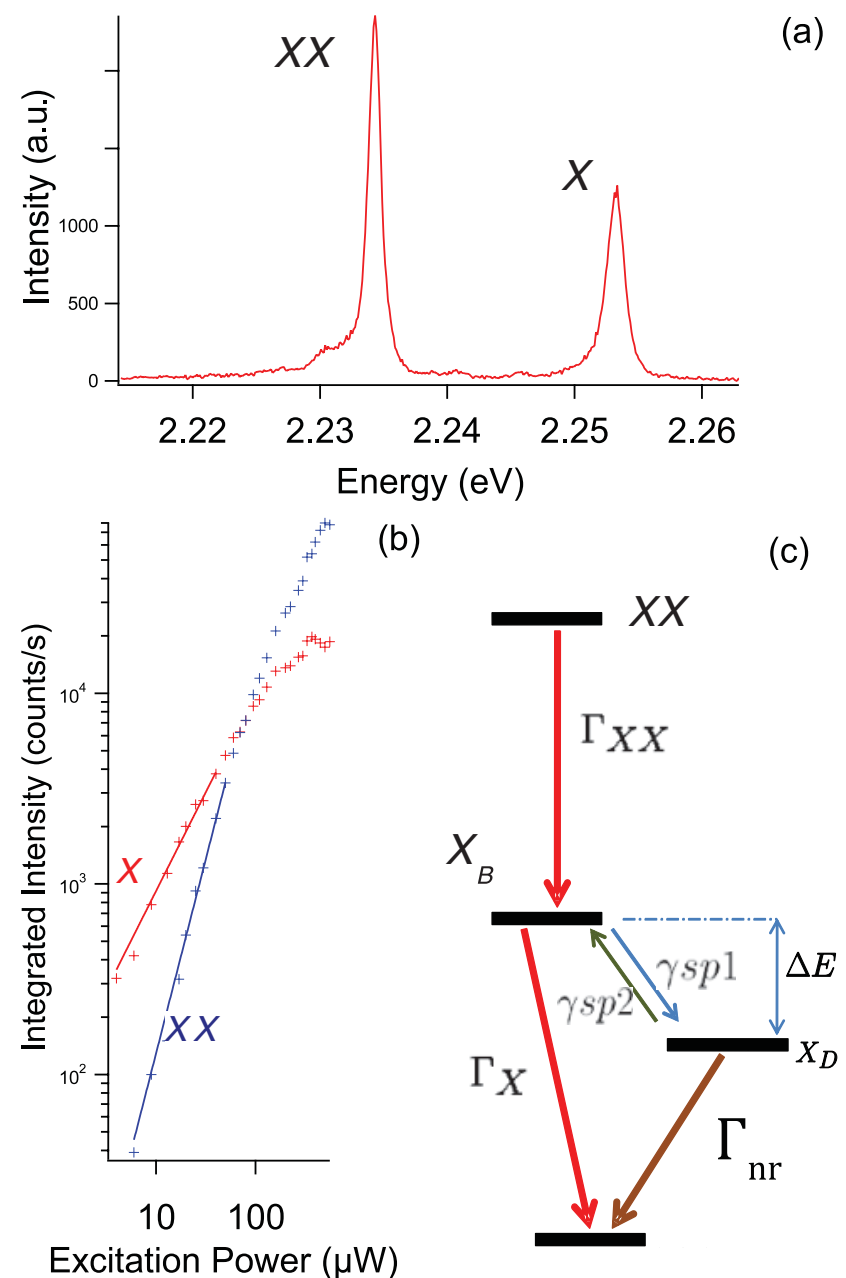

FIG. 1. (Color online) (a) Spectrum of a neutral QD at $T=4 \mathrm{~K}$. (b) Power dependence of the $X$ and $X X$ lines under pulsed excitation at $T=4 \mathrm{~K}$. (c) Level scheme and transition rates of a neutral QD.
QDs as $1 / \Gamma_{X}=0.50 \pm 0.05 \mathrm{~ns}, 1 / \Gamma_{X X}=0.30 \pm 0.05 \mathrm{~ns}$, and $1 / \Gamma_{\mathrm{nr}}=5.0 \pm 0.5 \mathrm{~ns}$, where the error is the dispersion amongst QDs. The measurement of the nonradiative decay rate $\Gamma_{\mathrm{nr}}$ is explained in the following paragraph. An example of power dependence under pulsed excitation is shown in Fig. 1(b) evidencing the linear and quadratic behavior of the $X$ and $X X$ line intensity, respectively. In all the investigated QDs, saturating intensity of the exciton emission is smaller than the biexciton, which is a signature of the strong influence of the dark exciton. ${ }^{24}$ When $\Delta E \gg k_{B} T$, dark excitons cannot transit back to the bright state, as there are no phonons available for such a spin flip and dark excitons are stored until recombining nonradiatively. As a result, the photoluminescence of the exciton is less intense than that of the biexciton at saturation. The biexciton luminescence is not affected by the presence of the DS, whereas the exciton BS has a large probability to decay to the DS and not produce any photon. As spin-flip rates here are of the same order of magnitude or larger than the BS radiative decay, exciton intensity is generally small compared to the biexciton.

Following the level scheme shown in Fig. 1, time-resolved exciton luminescence decays excited well below saturation exhibit biexponential dependency, the fitting of which gives access to the downward and upward spin-flip rates $\gamma_{\mathrm{sp} 1}$ and $\gamma_{\mathrm{sp} 2}$, and the nonradiative decay rate $\Gamma_{\mathrm{nr}}$ of the DS. The temperature dependency of $\gamma_{\mathrm{sp} 1}$ and $\gamma_{\mathrm{sp} 2}$ yields to the energy splitting $\Delta E$ between DS and BS. This is due to the fact that spin-flip transition rates depend on the spectral density of the acoustic phonons at the energy of the transition $\Delta E$ and on the phonon population that follows a Bose-Einstein distribution $N_{B}(\Delta E, T)=1 /\left[1-\exp \left(\Delta E / k_{B} T\right)\right]$ (see Refs. 25 and 20 for details). The spin-flip rates $\gamma_{\mathrm{sp} 1}$ (bright to dark) and $\gamma_{\mathrm{sp} 2}$ (dark to bright) are then written as

$$
\begin{aligned}
& \gamma_{\mathrm{sp} 1} \propto\left[N_{B}(\Delta E, T)+1\right] R(\Delta E), \\
& \gamma_{\mathrm{sp} 2} \propto N_{B}(\Delta E, T) R(\Delta E) .
\end{aligned}
$$

Figure 2 shows the spin-flip rates $\gamma_{\mathrm{sp} 1}$ and $\gamma_{\mathrm{sp} 2}$ versus temperature obtained after fitting the time-resolved luminescence

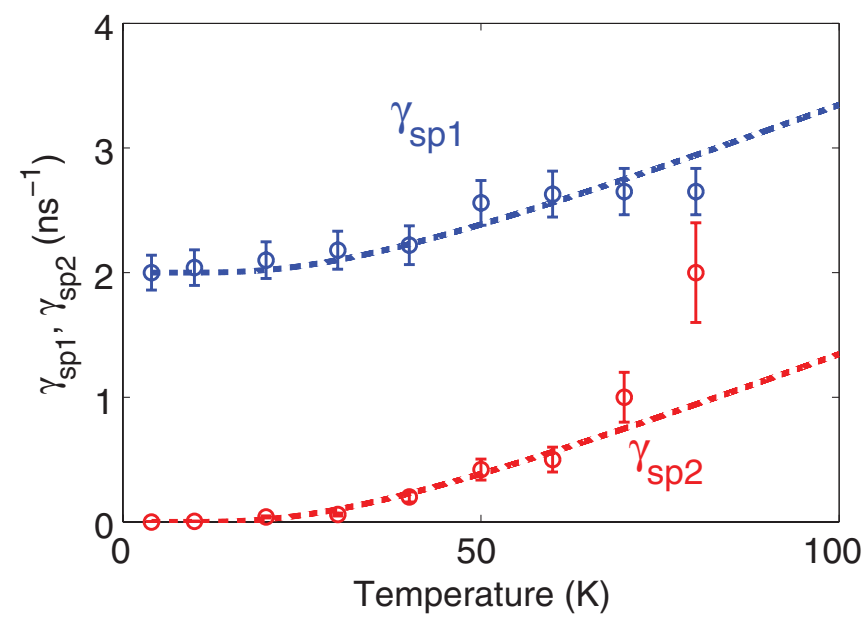

FIG. 2. (Color online) Transition rates $\gamma_{\mathrm{sp} 1}$ and $\gamma_{\mathrm{sp} 2}$ vs temperature. The dashed lines are the plots from the theoretical model following phonon population evolution with temperature [Eqs. (1) and (2)] with $\Delta E=7.2 \mathrm{meV}$. 


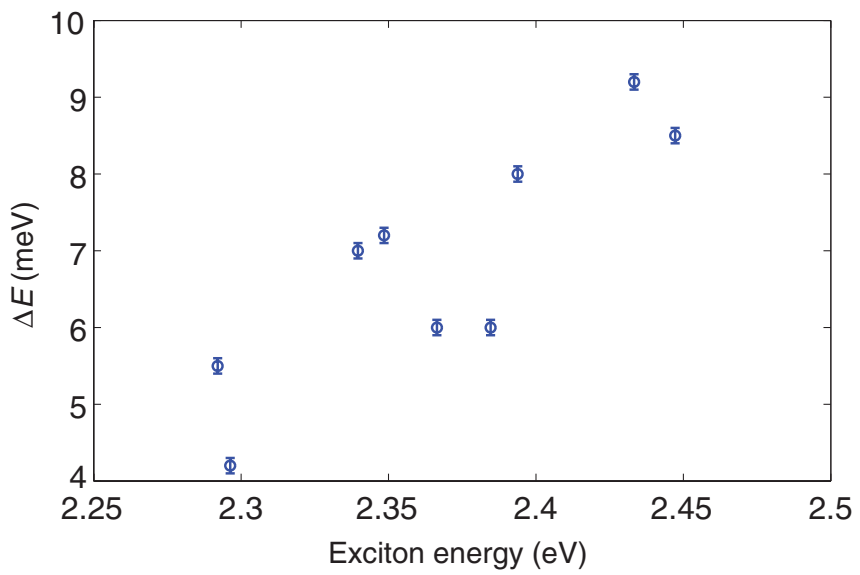

FIG. 3. (Color online) Increase of splitting energies $\Delta E$ between DS and BS measured on nine QDs as a function of their excitonic emission energy.

decay data (not shown). Note that the quantity $R(\Delta E)$ depends on the BS-DS energy splitting $\Delta E$, but also on the spatial extension and shape of the wave function of the exciton. ${ }^{30,31}$ The nonradiative decay rate $\Gamma_{\mathrm{nr}}$ is increasing with temperature according to an Arrhenius law with an activation energy of $E_{a}=30 \pm 5 \mathrm{meV}$.

At $T=0 \mathrm{~K}, N_{B}=0$, so that the spectral density $R(\Delta E) \propto$ $\gamma_{\mathrm{sp1}}(0 \mathrm{~K})$ represents the bright to dark state transition rate and $\gamma_{\mathrm{sp} 2}=0$. When the temperature is increased so that $k_{B} T \sim \Delta E$, the bright exciton state is repopulated. For each temperature, the transition rates $\gamma_{\mathrm{sp} 1}$ and $\gamma_{\mathrm{sp} 2}$ are extracted from the time-resolved luminescence and their evolution is fitted using the model based on the level scheme of Fig. 1(c) with $\Delta E$ as the fitting parameter.

This process is repeated on nine QDs. As shown in Fig. 3, measured splittings range from 4.2 to $9.2 \mathrm{meV}$. A clear trend is appearing: splittings measured for low-energy emission QDs are generally smaller compared to splittings measured for highenergy emission QDs. The latter correspond to small-size QDs, where electron and hole wave functions overlap very well, so that their exchange energy is large, leading to large $\Delta E$. Such relationships between QD sizes and energy splittings have been calculated for colloidal QDs. ${ }^{32}$ Change in composition of the $\mathrm{Cd}_{x} \mathrm{Zn}_{1-x}$ Se QD can also affect the emission energy and the value of $\Delta E$. Our measurements do not follow exactly a smooth law. The shape of the confinement has also a strong influence on the wave function forms, and consequently on their correlation function. For example, the prolate or oblate nature of the QD geometry appears to have an important effect. This explains why we obtained a cloud of experimental points following a general trend instead of a strict dependence.

\section{EFFICIENCY OF THE EXCITON-PHONON COUPLING}

In Fig. 4, we have plotted the transition rates from BS to DS measured at $4 \mathrm{~K}, \gamma_{\mathrm{sp} 1}(4 \mathrm{~K})$, versus energy splittings $\Delta E$ for nine different QDs. A clear enhancement of the transition rate for large $\Delta E$ can be observed. It can be also noted that the measured rates are comparable and even larger than the excitonic radiative rate. ${ }^{24}$ This is why the bright exciton is

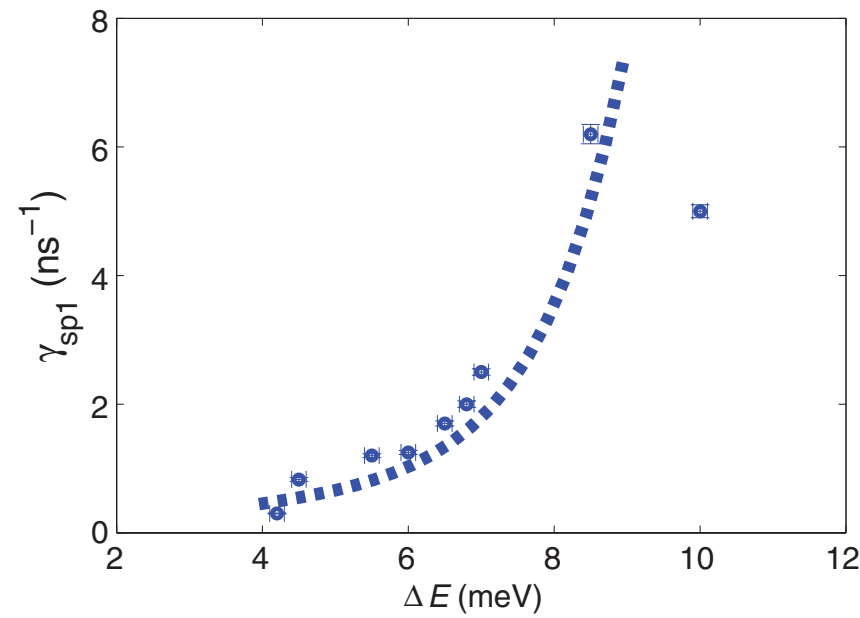

FIG. 4. (Color online) Transition rate from BS to DS $\gamma_{\mathrm{sp} 1}$ at $4 \mathrm{~K}$ plotted as a function of splitting energy $\Delta E$ between DS and BS for nine differents QDs. The dotted line is a guide for the eyes.

so depopulated compared to the biexciton in all the QDs investigated.

The results displayed in Fig. 4 indicate that large $\Delta E$ splittings lead to extremely fast depopulation of the bright state, suggesting that exciton-phonon interaction becomes more efficient with large $\Delta E$. As explained, larger splittings correspond to small QDs. Exciton coupling to phonons not only depends on the energy needed for the spin flip, but also on the QD size. However, size and $\Delta E$ are not independent parameters and induce opposite effects on the transition rates. In order to explain this nontrivial behavior, it is necessary to calculate the phonon spectral density as a function of QD size and energy splitting $\Delta E$ for each particular QD to obtain a general trend.

We shall only consider longitudinal-acoustical (LA) compression modes, since longitudinal-optical (LO) phonons have energies of about $30 \mathrm{meV}$ far above the dark-bright exciton splitting $\Delta E$. In the following, we will perform this calculation both in the three-dimensional (3D) and in the 1D case for the available phonon modes. The NW geometry is obviously somewhere in between these two extreme cases that will only give us a qualitative behavior.

We first consider the 3D situation. The phonons dispersion is bulklike, approximated by the Debye law $w(k)=c_{l} k$, with $c_{l}$ the sound speed for LA phonons in the semiconductor material. Piezoelectric interactions and Fröhlich longitudinal-optical (LO) phonon couplings are neglected. The exciton-phonon interaction is dominated by the hole-phonon interaction. ${ }^{26} \mathrm{We}$ therefore consider only the latter, whose Hamiltonian can be written, in the second quantization representation with respect to the carrier states, as

$$
H_{h}=\frac{1}{\sqrt{N}} \sum_{k n n^{\prime}} a_{n}^{\dagger} a_{n}^{\prime} f_{h, n n^{\prime}}(k)\left(b_{k}+b_{-k}\right)
$$

where $a_{n}^{\dagger}$ and $a_{n}$ are the hole creation and annihilation operators, and $b_{k}^{\dagger}$ and $b_{k}$ are LA phonon creation and annihilation operators. The index $n$ represents the excitation level of the 
hole, and $k$ the phonon mode. The coupling constant is defined as

$$
f_{h, n n^{\prime}}(k)=\sigma_{h} \sqrt{\frac{\hbar k}{2 \rho V c_{l}}} F_{n n^{\prime}}(k),
$$

where $\sigma_{h}$ is the deformation potential for holes, $V$ is the unitcell volume, and $\rho$ is the CdSe volumic mass. The quantity $F_{n n^{\prime}}(k)$ is a purely geometrical form factor given by ${ }^{27}$

$$
F_{n n^{\prime}}(k)=\int_{-\infty}^{\infty} d^{3} r \psi_{n}^{*}(r) e^{i k r} \psi_{n^{\prime}}(r)
$$

In order to evaluate the coupling constant for the lowest hole state $f_{11}(E)$, we consider the harmonic-oscillator potential ground state as the wave function of the hole:

$$
\psi(r)=\frac{1}{\pi^{3 / 4} l_{\perp} \sqrt{l_{z}}} \exp \left[-\frac{1}{2}\left(\frac{r_{\perp}}{l_{\perp}}\right)^{2}-\frac{1}{2}\left(\frac{z}{l_{z}}\right)^{2}\right],
$$

where $r_{\perp}$ is the position component in the $x y$ plane and $l_{\perp}, l_{z}$ are respectively the in-plane, and out-of-plane ( $z$ direction) localization widths. For this wave function, the form factor is easily found as

$$
F_{11}(k)=\exp \left[-\left(\frac{k_{\perp} l_{\perp}}{2}\right)^{2}-\left(\frac{k_{z} l_{z}}{2}\right)^{2}\right] .
$$

For the lowest hole state, the phonon spectral density in the QD is

$$
\begin{aligned}
R(E)= & \frac{1}{\hbar^{2}}\left[N_{B}(E)+1\right] \frac{1}{N} \sum_{k} f_{h, 11}(k) \\
& \times f_{h, 11}^{*}(k)[\delta(E-E(k))+\delta(E+E(k))] .
\end{aligned}
$$

After performing the summation over $k$ in the continuum limit, introducing the quadratic density of state of the phonons corresponding to the $3 \mathrm{D}$ case, the phonon spectral density is

$$
R(E)=R_{3 \mathrm{D}}(E)=R_{o} E^{3} g_{3 \mathrm{D}}(E),
$$

with

$$
R_{o}=\frac{\left(\sigma_{h}\right)^{2}}{8 \pi^{2} \hbar \rho c_{l}^{5}} .
$$

The quantity $R_{o}$ contains all material parameters. The cubic dependence is due to the quadratic phonon density of state, and the function $g_{3 \mathrm{D}}(E)$ is a function of the energy and of the geometrical parameters of the $\mathrm{QD},{ }^{33}$

$$
\begin{aligned}
g_{3 \mathrm{D}}(E) & =\int_{-\frac{\pi}{2}}^{\frac{\pi}{2}} \zeta \cos \zeta \exp \left[-\frac{\left(l_{\perp}\right)^{2} E^{2}}{2 \hbar^{2} c_{l}^{2}}\right. \\
& \left.\times\left(\cos ^{2} \zeta+\frac{l_{z}^{2}}{\left(l_{\perp}\right)^{2}} \sin ^{2} \zeta\right)\right] d \zeta,
\end{aligned}
$$

where $\zeta$ is the angle of the wave vector $k$ with respect to the normal to the $z$ direction.

Describing the coupling of the hole to phonons confined in a nanowire the same way as in a 3D semiconductor bulk matrix seems a rough approximation. Therefore, we also considered the $1 \mathrm{D}$ case in which the nanowire is taken as an infinitely thin monomode wave guide. The phonon density of state is constant and we consider that only the phonons propagating along the nanowire ( $z$ direction) can couple to the hole whose wave function is taken as $\psi_{h}(r) \propto \exp \left[-(1 / 2)\left(z / l_{z}\right)^{2}\right]$. The spectral density becomes

$$
R(E)=R_{1 \mathrm{D}}(E) \propto E g_{1 \mathrm{D}}(E),
$$

with

$$
g_{1 \mathrm{D}}(E)=\exp \left[-\frac{l_{z}^{2} E^{2}}{2 \hbar^{2} c_{l}^{2}}\right] .
$$

The phonon spectral density is linear with energy, but the geometrical factor $g_{1 \mathrm{D}}(E)$ has the same Gaussian energy dependence as $g_{3 \mathrm{D}}(E)$.

Since at $T=4 \mathrm{~K}, \gamma_{\mathrm{sp} 1}(4 \mathrm{~K}) \propto R(\Delta E)$, the $\mathrm{BS}$ to $\mathrm{DS}$ spin-flip rate evolution from dot to dot can be described by calculating the phonon spectral density for each QD. Three calculated 3D phonon spectral densities corresponding to three different $l_{z}$ values $\left(l_{z}=2,4,6 \mathrm{~nm}\right)$ are plotted in Fig. 5(a). Because of its increase with energy (linear for 1D, cubic for 3D), $R(\Delta E)$ corresponding to QDs with smaller $l_{z}$ have their maximum enhanced and shifted toward the higher energies. As a consequence, in small QDs, high-energy phonons couple more efficiently with the hole. We propose to (a)

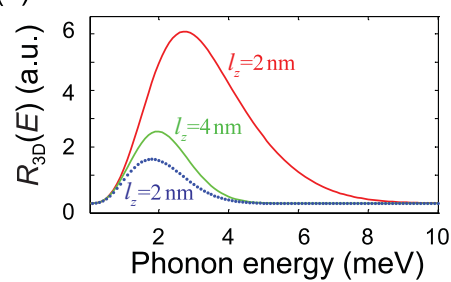

(c)

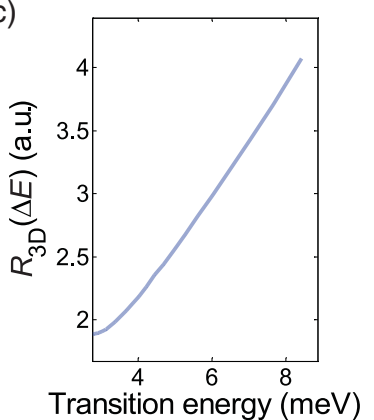

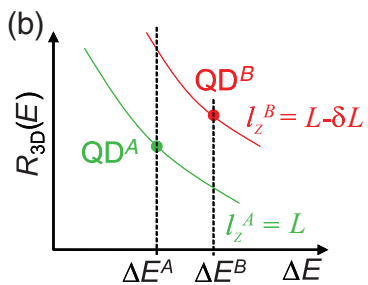

(d)

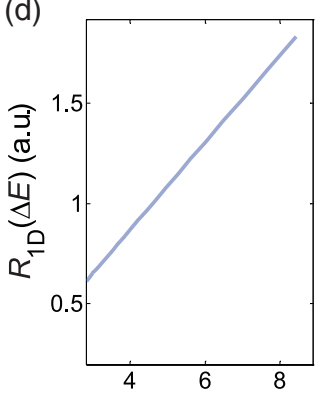

Transition energy (meV)
FIG. 5. (Color online) (a) 3D phonon spectral density calculated from Eq. (9) for three QD with different sizes along the $z$ direction (red solid line: $l_{z}=2 \mathrm{~nm}$; green dashed line: $l_{z}=4 \mathrm{~nm}$; blue dotted line: $l_{z}=6 \mathrm{~nm}$ ). Smaller QD $l_{z}$ dimension shifts the maximum of the coupling constant toward higher energies and enhances its relative value. (b) Zoom of the high-energy side of (a) for two quantum dots $\mathrm{QD}^{A}$ and $\mathrm{QD}^{B}$ with size $l_{z}^{A}>l_{z}^{B}$, which leads to bright-dark splittings $\Delta E^{A}<\Delta E^{B}$. When going from a big dot $\left(\mathrm{QD}^{A}\right)$ to a small dot $\left(\mathrm{QD}^{B}\right)$, the size effect alone leads to an increase of $R_{3 \mathrm{D}}$, but reduction of size comes with an increase of the bright-dark splitting, which reduces the final value of $R_{3 \mathrm{D}}$. (c) Calculated 3D phonon spectral density as a function of bright-dark splitting $\Delta E$. Here, each value $\Delta E$ corresponds to a different QD with a different size $l_{z}$ following Eq. (14). (d) Same as (c), but in the 1D case. Both (c) and (d) give a good qualitative description of the experimental results presented in Fig. 4. 
explain qualitatively the increase of the transition rate observed in Fig. 4 by this size effect. ${ }^{34}$

The phonon spectral density $R(\Delta E)$ of a QD with brightdark splitting $\Delta E$ depends on the QD size $l_{z}$ directly and via the energy splitting $\Delta E$ size dependency. We determine the relation between $l_{z}$ and $\Delta E$ by evaluating the short-range hole-electron exchange energy in the QD:

$$
\Delta E=\Delta E^{3 d} \frac{1}{\left|\varphi^{3 d}(0)\right|^{2}} \int\left|\psi_{e}(r)\right|^{2}\left|\psi_{h}(r)\right|^{2} d^{3} r,
$$

where $\psi_{e / h}(r)$ are the electron/hole wave functions, $\Delta E^{3 d}$ is the exchange energy in the bulk material $\mathrm{ZnCdSe}^{35}$ $\left(\Delta E^{3 d}=0.19 \mathrm{meV}\right.$, for a $\mathrm{Cd}_{0.5} \mathrm{Zn}_{0.5}$ Se composition of the QD as measured in high-resolution TEM experiments ${ }^{29}$ ), and $\left|\varphi^{3 d}(0)\right|^{2}=1 / \pi a_{B}^{* 3}$, with $a_{B}^{*}$ the Bohr radius of the free exciton. To match the measured values of $\Delta E$ (from 4 to $9 \mathrm{meV}$ ), we set the lateral confinement parameter $l_{\perp}$ at $4 \mathrm{~nm}$. With $l_{z}$ linked to a corresponding $\Delta E$, we make $l_{z}$ vary from 2 to $6 \mathrm{~nm}$ and we calculate $R(\Delta E)$.

The results, for the $3 \mathrm{D}$ and $1 \mathrm{D}$ cases described above, are plotted in Figs. 5(c) and 5(d). Because of the simplicity of the considered exciton wave function, the aim of the calculation is neither to fit the experimental data of Fig. 4 nor to obtain a quantitative estimation of the transition rates. However, we can show that the phonon spectral density is increasing for QD with large $\Delta E$ in both cases, which is not a trivial result, as the cutoff imposed by the dimensions of the QD makes this efficiency vanish for higher-energy phonons. Indeed, as shown in Fig. 5 (b), reduction of QD size leads to an increase of the phonon spectral density $R_{3 \mathrm{D}}$, but reduction of size means bright-dark splitting $\Delta E$ increase, which tends in turn to decrease $R_{3 \mathrm{D}}$. As a result, it turns out that the phonon spectral density $R_{3 \mathrm{D}}$ does increase with $\Delta E$, as observed experimentally in Fig. 4. The $1 \mathrm{D}$ and 3D situations considered here are extreme cases and we can expect a real nanowire geometry to follow an intermediate behavior for the hole phonon coupling.

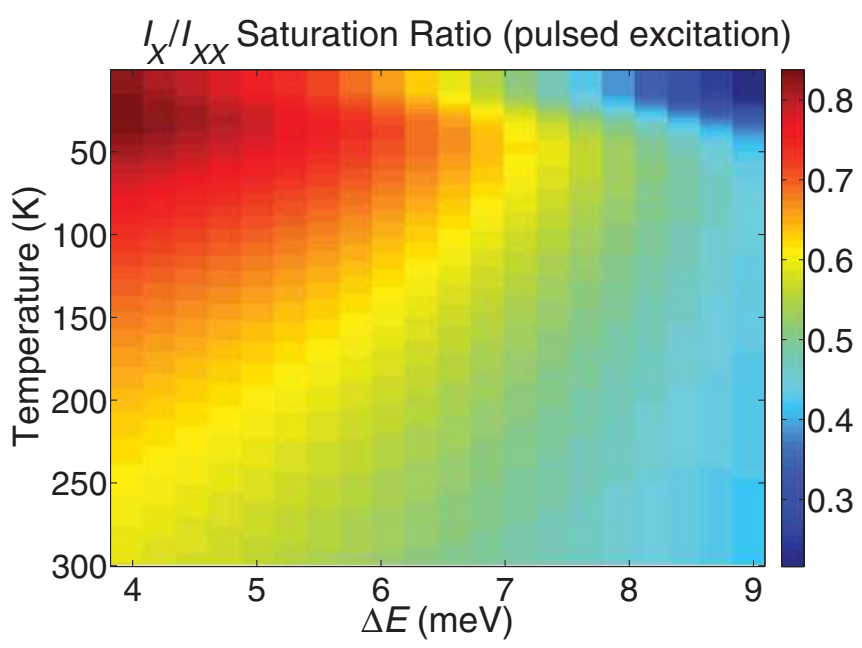

FIG. 6. (Color online) Calculated saturation ratio $I_{X} / I_{X X}$ under pulsed excitation as a function of $\Delta E$ and temperature. The color legend represents $I_{X} / I_{X X}$ saturation ratio. The cold colors show low exciton intensity and the hot color high intensity.

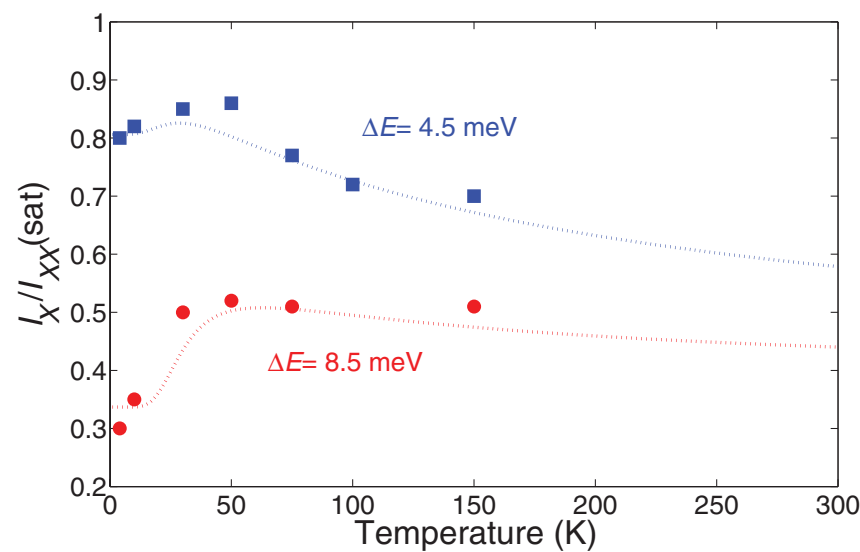

FIG. 7. (Color online) Experimental exciton-biexciton saturation ratio under pulsed excitation vs temperature for two QDs with respectively $\Delta E=4.5 \mathrm{meV}$ (blue squares) and $\Delta E=8.5 \mathrm{meV}$ (red circles). Dashed lines are vertical cut (fixed $\Delta E$ ) from the model used in Fig. 6.

\section{EXCITON LUMINESCENCE INTENSITY}

This increase of the exciton-phonon coupling efficiency with energy splitting has some consequences on the temperature-dependent exciton-biexciton saturation ratio under pulsed excitation. This calculated ratio is represented in Fig. 6 as a function of temperature and energy splitting $\Delta E$. We used the level scheme presented in Fig. 1 and the values of the transition rates given in Sec. III. The relation between $\gamma_{\mathrm{sp} 1}(4 \mathrm{~K})$ and $\Delta E$ is taken from the function used as a guide for the eyes in Fig. 4. We also considered the increase of the dark exciton nonradiative recombination $\Gamma_{\mathrm{nr}}(T)$ with temperature following an Arrhenius law $\Gamma_{\mathrm{nr}}(T) \sim \exp \left(-E_{a} / k_{B} T\right)$, with an activation energy $E_{a}$. For the two QDs studied in Fig. 7, we have measured $E_{a}=30 \pm 5 \mathrm{meV}$ and we have taken $E_{a}=$ $30 \mathrm{meV}$ in the model used in Figs. 6 and 7. The particularity here is that the DS lifetime at $T=4 \mathrm{~K}\left(1 / \Gamma_{\mathrm{nr}}=5 \mathrm{~ns}\right)$ is very short compared to values reported in other systems (up to $1 \mu \mathrm{s}^{25}$ ) and is of the same order of magnitude as the exciton radiative lifetime when temperature is raised up to only several tens of $\mathrm{K}$. A characteristic temperature behavior is shown in Fig. 6. The exciton intensity is very small at low temperature (particularly in the high splitting region), increases as temperature is increased up to $T=50 \mathrm{~K}$, and finally decreases with higher temperatures. The effect of repopulation of the bright exciton due to the DS to BS spin flip is compensated and overwhelmed by the exciton population loss caused by the faster dark exciton nonradiative recombination. As shown in Fig. 7, representing the excitonbiexciton ratio versus temperature for two different QDs with different measured $\Delta E$ (4.5 and $8.5 \mathrm{meV})$, this exciton line intensity decrease is more sensitive for large $\Delta E$. However, at $300 \mathrm{~K}$, the bright exciton is still less luminescent for large splittings. It can be noted that the model fits rather well the experimental data.

\section{CONCLUSION}

In summary, the exciton-phonon coupling efficiency is highly influenced by the QD size. This can explain the 
observed enhancement of the bright to dark spin-flip rate with increasing splitting energy. As a result, the saturation intensity of exciton transition is a lot weaker than the biexciton one. This effect is all the more important as the QD exchange splitting $\Delta E$ is larger. The domination of the biexcitonic line over the excitonic line is preserved at high temperature despite the bright state repopulation owing to the temperature-induced loss of excitonic population through the nonradiative dark exciton recombination. This exciton weakness could suggest the use of the biexciton line for high-temperature single-photon sources. In that case, the contamination by the exciton line could be reduced when the lines are broadened at high temperature.

\section{ACKNOWLEDGMENTS}

We acknowledge support from the French National Research Agency (ANR) through the Nanoscience and Nanotechnology Program (Project BONAFO ANR-08-NANO-031-01) that provided a research fellowship for M.d.H. M.E.J. acknowledges financial support from the Nanosciences Foundation "Nanosciences, aux limites de la nanoélectronique" (RTRA).
${ }^{1}$ X. Duan, Y. Huang, Y. Cui, J. Wang, and C. M. Lieber, Nature (London) 409, 66 (2001).

${ }^{2}$ W. Lu and C. M. Lieber, Nat. Mater. 6, 841 (2007).

${ }^{3}$ C. Thelander, T. Martensson, M. T. Björk, B. J. Ohlsson, M. W. Larsson, L. R. Wallenberg, and L. Samuelson, Appl. Phys. Lett. 83, 2052 (2003).

${ }^{4}$ R. Könenkamp, R. C. Word, and C. Schlegel, Appl. Phys. Lett. 85, 6004 (2004).

${ }^{5}$ H. M. Kim, Y. H. Cho, H. Lee, S. I. Kim, S. R. Ryu, D. Y. Kim, T. W. Kang, and K. S. Chung, Nano Lett. 4, 1059 (2004).

${ }^{6}$ X. Duan, Y. Huang, R. Agarwal, and C. M. Lieber, Nature (London) 421, 241 (2003).

${ }^{7}$ A. I. Hochbaum, R. Chen, R. D. Delgado, W. Liang, E. C. Garnett, M. Najarian, A. Majumdar, and P. Yang, Nature (London) 451, 163 (2008).

${ }^{8}$ Y. Cui, Q. Wei, H. Park, and C. M. Lieber, Science 293, 1289 (2001).

${ }^{9}$ M. S. Gudiksen, L. Lauhon, J. Wang, D. C. Smith, and C. M. Lieber, Nature (London) 415, 617 (2002).

${ }^{10}$ M. T. Björk, B. J. Ohlsson, T. Sass, A. I. Persson, C. Thelander, M. H. Magnusson, K. Deppert, L. R. Wallenberg, and L. Samuelson, Nano Lett. 2, 87 (2002); Appl. Phys. Lett. 80, 1058 (2002).

${ }^{11}$ C. Yang, Z. Zhong, and C. M. Lieber, Science 310, 1304 (2005).

${ }^{12}$ P. Michler, A. Kiraz, C. Becher, W. V. Schoenfeld, P. M. Petroff, L. Zang, E. Hu, and A. Imamoglu, Science 290, 2282 (2000).

${ }^{13}$ E. Moreau, I. Robert, J.-M. Gérard, I. Abram, L. Manin, and V. Thierry-Mieg, Appl. Phys. Lett. 79, 2865 (2001).

${ }^{14}$ C. Santori, D. Fattal, J. Vučković, G. S. Solomon, and Y. Yamamoto, Nature (London) 419, 594 (2002).

${ }^{15}$ W. H. Chang, W. Y. Chen, H. S. Chang, T. P. Hsieh, J. I. Chyi, and T. M. Hsu, Phys. Rev. Lett. 96, 117401 (2006).

${ }^{16}$ J. Claudon, J. Bleuse, N. S. Malik, M. Bazin, P. Jaffrennou, N. Gregersen, C. Sauvan, P. Lalanne, and J.-M. Gérard, Nat. Photon. 4, 174 (2010).

${ }^{17}$ M. T. Borgström, V. Zwiller, E. Müller, and A. Imamoglu, Nano Lett. 5, 1439 (2005).

${ }^{18}$ A. Tribu, G. Sallen, T. Aichele, R. André, C. Bougerol, S. Tatarenko, J.-Ph. Poizat, and K. Kheng, Nano Lett. 8, 4326 (2008).
${ }^{19}$ V. D. Kulakovskii, G. Bacher, R. Weigand, T. Kümmell, A. Forchel, E. Borovitskaya, K. Leonardi, and D. Hommel, Phys. Rev. Lett. 82, 1780 (1999); L. Besombes, K. Kheng, and D. Martrou, ibid. 85, 425 (2000).

${ }^{20}$ G. Sallen, A. Tribu, T. Aichele, R. André, L. Besombes, C. Bougerol, S. Tatarenko, K. Kheng, and J.-Ph. Poizat, Phys. Rev. B 80, 085310 (2009).

${ }^{21}$ Y. Chen, B. Gil, P. Lefebvre, and H. Mathieu, Phys. Rev. B 37, 6429 (1988).

${ }^{22}$ Y. Chen, Phys. Rev. B 41, 10604 (1990).

${ }^{23}$ M. Nirmal, D. J. Norris, M. Kuno, M. G. Bawendi, Al. L. Efros, and M. Rosen, Phys. Rev. Lett. 75, 3728 (1995).

${ }^{24}$ M. Reischle, G. J. Beirne, R. Rossbach, M. Jetter, and P. Michler, Phys. Rev. Lett. 101, 146402 (2008).

${ }^{25}$ O. Labeau, P. Tamarat, and B. Lounis, Phys. Rev. Lett. 90, 257404 (2003).

${ }^{26}$ L. M. Woods, T. L. Reinecke, and R. Kotlyar, Phys. Rev. B 69, 125330 (2004).

${ }^{27}$ A. Grodecka, L. Jacak, P. Machnikowski, and K. Roszak, e-print arXiv:cond-mat/0404364 (2004)

${ }^{28}$ M. Den Hertog, M. Elouneg-Jamroz, E. Bellet-Amalric, S. Bounouar, C. Bougerol, R. André, Y. Genuist, J.-Ph. Poizat, K. Kheng, and S. Tatarenko, J. Cryst. Growth 323, 330 (2011).

${ }^{29}$ M. Den Hertog, M. Elouneg-Jamroz, E. Bellet-Amalric, S. Bounouar, C. Bougerol, R. André, Y. Genuist, J.-Ph. Poizat, K. Kheng, and S. Tatarenko, J. Appl. Phys. 110, 034318 (2011).

${ }^{30}$ L. Besombes, K. Kheng, L. Marsal, and H. Mariette, Phys. Rev. B 63, 155307 (2001).

${ }^{31}$ T. Takagahara, Phys. Rev. B 60, 2638 (1999).

${ }^{32}$ Al. L. Efros, M. Rosen, M. Kuno, M. Nirmal, D. J. Norris, and M. Bawendi, Phys. Rev. B 54, 4843 (1996).

${ }^{33}$ A. Grodecka, C. Weber, P. Machnikowski, and A. Knorr, Phys. Rev. B 76, 205305 (2007).

${ }^{34}$ E. Tsitsishvili and H. Kalt, Phys. Rev. B 82, 195315 (2010); Y.-H. Liao, J. I. Climente, and S.-J. Cheng, ibid. 83, 165317 (2011).

${ }^{35}$ J. Puls, F. Henneberger, M. Rabe, and A. Siarkos, J. Cryst. Growth 184/185, 787 (1998). 